\title{
Structure of Starch-ionic Surfactant Complexes Studied by Ternary Phase, XRD and Scanning Electron Microscopy
}

\author{
Md. MOHSIN HOSSAIN, Md. IBRAHIM H. MONDAL and Md. RAIHAN SHARIF \\ Polymer and Textile Research Lab, Department of Applied Chemistry \\ and Chemical Engineering, Rajshahi University, Bangladesh. \\ ${ }^{*}$ Corresponding author E-mail: mihmondal@yahoo.com
}

http://dx.doi.org/10.13005/ojc/300109

(Received: January 19, 2014; Accepted: March 05, 2014)

\begin{abstract}
The structure of Iyotropic liquid crystallineorgel like phases formedin starch/surfactant (cationicoranionic)/ water ternary phase systems in the temperature range of $25-85^{\circ}$ Chas been investigated by XRD, SEM and FT-IR techniques. The ionic surfactants were cetyltrimethyl ammonium bromide (CTAB), sodiumd odecyl sulfate (SDS). The phases were in equilibrium with aqueous solutions at $60^{\circ} \mathrm{C}$ and contained $15-25 \mathrm{wt} \%$ starch and $10-30 \mathrm{wt} \%$ surfactant, depending on the charge density of the starch and the chain length of the surfactant. The phases consist of starch / surfactant aggregates arranged in long-range structures similar tolyotropicmeso phases formed by the pure ionic surfactant salone, but they separate from aqueous solutions at much lower surfactant concentrations. When the charge density of the starchis low or the surfactant hydrocarbon chain is short, the characteristic cubicor hexagonal phases are formed. As expected, the formation of lamellar phaseis promoted by increasing these parameters. Temperature affects the stability of the phases and their structure. At high temperatures the long-range order breaks down, and the phases are akin to concentrated micellar starch / ionic surfactant solutions.
\end{abstract}

Key words: Starch, ionic surfactant, starch-ionic surfactant complexes, ternary phase diagram.

\section{INTRODUCTION}

The research understanding of polymer/ surfactant interactions in aqueous solution has been summarized in several reviews ${ }^{1-5}$. Many investigations have focused on aggregate structure in dilute solution, i.e., system in which phase separation does not occur. The general picture emerging from these studies is that in dilute solution the surfactant molecules adsorb to polymer chains as micellar or micelle-like clusters. A general phenomenon in system of polyelectrolyte and oppositely charged surfactant is that complexes of these components separate as a water-swollen phase in equilibrium with very dilute aqueous solution. Generally, the rich phase behavior of surfactants in water is also characteristic of polyelectrolyte-surfactant complexes in contact with water. Harada and Nozakura ${ }^{6}$ described the formation of layered structures in polyvinyl sulfate (PVS)/ cetyltrimethylammonium 
bromide and 1-4-ionene /sodium dodecyl sulfate (SDS) systems. More extensive studies of $x, y$-ionene / SDS andPVS/n-alkyl pyridinium surfactants systems have recently been reported by Chen et al. ${ }^{7}$, Kim et al. ${ }^{8}$, Kabanov et al. ${ }^{9}$ and Khandurina et al. ${ }^{10}$, Dembo et al. ${ }^{11}$ and Rogacheva et al. ${ }^{12}$ observed the complex lamellar structures of sodium poly(acrylate)gel and alkyltrimethyl ammonium bromides. Hansson and Langmuir $^{12}$ found that a cubic phase was formed by sodiumpoly- (acrylate) and dodecyltrimethyl ammonium bromide. llekti et al. ${ }^{14}$ studiedsodium poly (acrylate)/ cetyltrimethyl ammonium bromide complexes and found that they form hexagonal structures, and that the ionic compositions of the concentrated and dilute phases have significant effect on the phase behavior. Kosmella et al., ${ }^{15}$ and Ruppelt et al. ${ }^{16}$ found that the intercalation of sodiumpoly (acrylate) into meso phases formed by alkyltrimethyl ammonium bromides induced some disordering, but did not substantially affect the phase structures. Very recently Zhou et al. ${ }^{17}$ observed long-range order in complexes of poly(sodium methacrylate-co-N-isopropyl-acrylamide) and poly (styrenesulfonate) with tetra decylhexadecyl, ordodecyltrimethyl ammonium bromide. Zhou et al. ${ }^{18}$ discussedacharacteristicofthe complexesin this investigation is that they contain a very hydrophilic carbohydrate polymer associated with oppositely charged surfactants. Thus, we expect the predominant driving forces for the self-assembly of surfactant molecules in these complexes to be hydrophobic interactions between the hydrocarbon chains of the surfactant and electrostatic interactions between charged segments of the polymer and the head groupsofthe surfactant. Previously, we have reported on interactions and rheological properties of the complex phase in these systems and the same was suggested by Merta and Stenius ${ }^{19}$ that the irgoalis to evaluate the structure of the phases in equilibrium with very dilute aqueous solution. Goddard and Hannan ${ }^{20}$ investigated the interactions between surfactants with cationized cellulose and also the rheology of the complexes by Goddard and Hannan ${ }^{21}$ but to the present study isthefirstinvestigationreportof starch/ionic surfactantsystemsforreal structure identification through XRD, SEM and ternary phase. An investigation of the structure of starchsurfactant complexes produced by intermolecular $\mathrm{H}$-bonding is very important for the clarification of the mechanism of formation of the complexation in between starch - surfactant interaction. However, only a few studies relating to the structure of the complexation have been reported to date. X-ray diffraction patterns of the insoluble residues of potato starch with monoglycerides or SDS, CTAB has showed strong surfactant - amylose complexes at $80^{\circ} \mathrm{C}$ temperature. These complexes at lower temperature showed that surfactants entered the starchgranules and formed complexes with the amylose at $95^{\circ} \mathrm{C}$. The use of surfactants in starch based productsis increasing because the irability to retard firming and retrogradation. The interaction between starch and surfactant was explained by earlier workers ${ }^{22,23}$ and the absorption of surfactant occurs on the surfaces of the starchgranules. Later research showed, however, that surface active agent for inclusion compounds or complexes with amylose, $\mathrm{X}$-ray diffraction patterns of all surfactant complexes have the so called V-amylose pattern supported by Zobel ${ }^{24}$. Osman et al. ${ }^{25}$ studied the ability of certain emulsifiers to complex with the amylose fraction of starch, evaluating them by their interference with the binding of iodine by amylase which showed ${ }^{26}$ that the ability of monoglycerides to form complexes with amylose dependent onthe physical form of the surfactant.KrogandNybo-Jensin ${ }^{27}$ also reported on the amylose complexing ability of several emulsifiers and found that distilled monoglycerides had the best complexing ability among ionic surfactants and that sodiums tearoyllactylate and calcium stearoyllactylate were best among the ion icones. The purpose of our study was to investigate starch- surfactant interaction by the measurement of crystalinity and the dissociation of thea mylosesurfactant complexes at definite temperature using XRD, SEM and FT-IRanalyses.

\section{EXPERIMENTAL}

\section{Materials}

This product was purchased fromUNICHEM, China. The degree of substitution (DS) was 0.80 . The starch was dissolved by heating the starch / water mixture in an autoclave at $120^{\circ} \mathrm{C}$ for 30 $\min$. All solutions were prepared atleast $24 \mathrm{~h}$ before measurements were performed.

The water was ion exchanged and distilled. Its conductivity and reduced viscosity were $2.0 \mu \mathrm{s}$ and $4.0 \mathrm{dm}^{3} / \mathrm{mol}$, and its surface tension was 71.5 
$\pm 0.5 \mathrm{mN} / \mathrm{mat} 30^{\circ} \mathrm{C}$. Surfactants and all other chemicals were of analytical grade and were used without further purification.

\section{Sample preparation for SEM}

Weighted amounts of starch, surfactants and distilled water were added to tightly closed test tubes. The tubes were equilibrated by continuously turning them over in a thermostat at $333 \mathrm{~K}$ (for starch/ SDS or CTAB the temperature was 298K) for 7 days. This resulted in the formation as a complex phase dispersed in aqueous solution. The charge neutrality of the complexes was verified by measuring the electrophoretic mobility of the dispersed particles. The solution and complex phases were separated by centrifugation for $30 \mathrm{~min}$ at $1600 \mathrm{~g}$. After centrifugation, samples were again allowed to equilibrate at the same temperature for 7 days. Then the phases were separated by careful decantation of the solution. The dry content of the complex phase was determined by weighing. Then the sample was analyzed by Scanning Electron Microgram.

\section{Sample preparation for FT-IR}

An FT-IR spectrum of dried sample was obtained with SHIMADZU IR-470 infra-red spectrophotometer. After drying the sample with KBrpellate at room temperature, it was again dried at $60^{\circ} \mathrm{C}$ for $1 \mathrm{~h}$ under reduced pressure and then its individual IR spectra were measured. In case of sample crystalline (Tween-20) IR spactra was measured on the Teflon plate at controlled temperature and pressure.

\section{Sample preparation for XRD}

For the preparation of starch sample, the dried sample is saturated with water by stirring repeatedly with a glass rod. This step is performed by $2 \mathrm{~g}$ of starch in $100 \mathrm{~mL}$ of hot distilled water and stirred at room temperature for $1 \mathrm{~h}$, centrifuging the suspension and decanting the supernatant solutions. This process is repeated three times. Then the starch solution is dried in oven at temperature $80^{\circ} \mathrm{C}$. After $2 \mathrm{~h}$ drying, the powder sample was kept in a sealed bottle. The syntheses of cationic surfactants were undertaken by the following procedure: $2 \mathrm{~g}$ of cetyltrimethylammonium bromide (CTAB) was first dispersed in $100 \mathrm{~mL}$ of deionized water andthen under mechanical stirring for about 1h. A pre-dissolved starch solution of same amount was slowly added to the suspension at $80^{\circ} \mathrm{C}$. The reaction mixtures were stirred for $1 \mathrm{~h}$ at $80^{\circ} \mathrm{C}$ using mechanical stirring. After proper drying the powder sample was kept in a sealed bottle and stored in a vacuum desiccator. Starch powders to be used for X-ray diffraction (XRD) measurements were equilibrated in a desiccators containing saturated solutions of $\mathrm{K}_{2} \mathrm{CO}_{3}$ at $20-22^{\circ} \mathrm{C}$. Under these conditions, the relative humidity $(\mathrm{RH})$ at $20^{\circ} \mathrm{C}$ was $44 \%$ and the final water content of potato starches was $15 \%$. Wet starch powder (from potato) for XRD measurements was produced by first equilibrating the starch excess water. The starch suspension was then centrifuged and the supernatant removed. The starch precipitate appeared as a hard wet powder that was slightly moremoist at the top. This moisture was dried with tissue paper. The wet starch powder had a water content of $49 \%$. It was apparent that the proportion of water was slightly overestimated, because the precipitated starch granules would have a small amount of free space between them, which would be filled with free water. This overestimation, however, can be considered to be very small as the granules in the precipitate were closely packed. Since the water content within the crystallites is fixed near about $24 \%$ the proportion of water in the amorphous part of starch can be estimated at 55$60 \%$. It was assumed that total crystallinity of starch and complexes crystallinity were within the range $25-40 \%$ and $40 / 60-50 / 50$ respectively.

\section{Samplepreparationforternaryphase}

An equilateral triangular wooden frame consists of 228 holes. For the development of ternary phase diagrams, the sample components were taken in to the test tube by varying composition in such away that the total composition remains $100 \%$. The components were added by varying weight by volumes. The samples were prepared by varying $5 \%$ composition of two components simultaneously keeping the third component constant alternatively in a testtube. The open end of the test tube was then closed with rubber corkin such away that the vapour comes out and enters in to the test tube, here the cork react with the sample. The samples were then shaken for well mixing of the components and placed in to the diagram according to the composition and mark. The openend of the test tube was then closed tightly with cork, so that this remains no leakage or the lower part of the cork did not touch 
the sample solution and these samples were then left to equilibrate in a thermost at box at $30^{\circ} \mathrm{C}$ for atleast ten days and shaken from time to time. The equilibrium was established with in this period.

\section{Scannning Electron Microscopic Examination Procedure for sample determination}

Scanning Electron Microscope (SEM) of potato starch, surfactant sample and starchsurfactant complexes samples were examined according to Atichokudomchai et al., ${ }^{27}$. All the sampleswere dried before examined. The excess sample was removed and place in a fine coater for gold coating for $150 \mathrm{sec}$. The coated sample was placed in a sample chamber in the SEM. The sample was examined at magnification of $2,500 \times$ and 6,000 $x$ with the accelerating voltage of $10 \mathrm{kV}$.

\section{SEM analysis procedure}

All samples were analyzed using a Scanning Electron Microscope with an Oxford INCA X-sight EDXA (Energy Dispersive X-Ray Analysis system). The SEM is a high-performance, Scanning Electron Microscopy with a high resolution of $3.0 \mathrm{~nm}$. The low vacuum mode allows for observation of specimens which cannot be viewed at high vacuum due to excessive water content or due to a non- conductive surface. SEM analysis can be used to examine surface features, textures and particles that are too small to see with standard optical microscopes.

Effect of adding surfactant (SDS, CTAB) on the starch structure

XRD was used to examine the type and extent of complexity resulting from cooling of the starch paste in the presence of SDS or CTAB. Increased relative intensity would generallyr eflecta greater degree of complexity. With out surfactants, control starch showed a typical A-type pattern ${ }^{28}$ (Fig.1a). With the addition of either of the surfactants, potato starch exhibited a typical V-type pattern after heating and cooling (Figs.1b and 1c). Similar result has been reported by Ghiasi et al.,29. WhenTangand Copeland ${ }^{30}$ found that mixed stearic acid and wheat starch had peaks at $, 2 \theta=7.4^{\circ}, 12.7^{\circ}$ and $19.8^{\circ}$. Similar V-type pattern was produced for starch with SDS in the present study. Results of the present study found that patterns of starch/CTAB had slightly greater relative intensity than did the patterns of starch plus SDS supported by Copeland et al., ${ }^{31}$. In the starch systems, XRD patterns in the presence of either surfactant were identical with respect to the type of crystalline structure. Like potato starch, diffraction patterns of SDS plus either starch (Fig. 1b) had a slightly greater intensity than did the diffraction patterns of either starch plus SDS (Fig. 1c). Unlike potato starch, diffraction peaks were not as prominent with either CTAB.With the addition of surfactants, potato starch and wheat flour produced a V-type diffraction pattern, while native wheat starch and flour had a typical A-type pattern similar to results reported by Abdel-Aal et al., ${ }^{32}$.

\section{RESULTS ANDDISCUSSION}

When starch was heated in excess water at75 and $95^{\circ} \mathrm{C}$, thesolublepartwasremovedandthe insoluble part was freeze-dried. X-raydiffraction patterns of the insoluble residues are presented in Fig. 1. The sample heated at $75^{\circ} \mathrm{C}$ (Fig.1b) showed a low degree of crystallinity, and the sample heated at $95^{\circ} \mathrm{C}$ (Fig.1a) was essentially non crystalline which Ghiasi et al..$^{28}$ showed at $75^{\circ} \mathrm{C}$ only $5.9 \%$ of the starch, mainly for amylose. There fore, at that temperature, even the major part of the amylose was still associated with the granules and the organization of the starch granule had changed. Considerable controversy exists concerning which components amyloseor amylopectin is responsible for the crystallinity of $\operatorname{starch}^{33-36}$. According to polymer physics, crystallities can occur whenever chain segments happen to be parallel in lattice positions. There fore, starch crystallities can form through hydrogen bonding between linear fraction and segments of the branched fraction which was supported by Caesar and Cushing ${ }^{37}$ [1971]. X-ray diffraction patterns of the in soluble residue of the starch obtained from heated starch to different temperatures with SDS is shown in the Fig.1c. With one exception $\left(95^{\circ} \mathrm{C}\right)$, the sample all showed the surfactant-amylose complex pattern, as indicated by the strong d-spacing at 4.4 and 6.8 Aoat $950 \mathrm{C}$, thestarch-SDS complex was no longer observed. Loss of the complex at $95^{\circ} \mathrm{C}$ helps explain why SDS did not prevent leaching of soluble from starch at high temperatures. Ghiasi et al..$^{29}$ and Gray and Schoch ${ }^{38}$ found that SDS was not effective in decreasing solubility of starches at temperatures of $85^{\circ} \mathrm{C}$ and above. X-ray diffraction patterns of soluble isolated from starch and surfactant treated starch heated at $95^{\circ} \mathrm{C}$ are shown in Fig.1C. X-raydiffraction patterns 
were primarily V-hydrate complex, asi ndicated by third-spacings marked by Zobel ${ }^{24}$. The existence of amyloseasa V-hydrate complex has been reported by Yamashita ${ }^{39}$. Original XRD Figs. 2, 3 and 4 has been given below for better understanding starch, SDS, CTAB complexes as variation of concentration.

From Figs. 5 and 6, It is seen that the surface of SEM is homogeneous but it is cleared to identify that the reacted starch-SDS complexes (Fig.7) had a lot of ruptured surfaces in the magnification area. Here it was also identified that the smoother surface exists in the starch and SDS SEM images only. So it is clearly decided that obviously interaction may occurred in the starch-SDS complexes. so, in complexes there is a change in structural configuration not only this in XRD images from Figs. 3 and 4 comparison with only starch morphology the changes are obtain from here that crystalinity varies from different angles and intensity analysis.

In summary, it can be concluded that liquid crystalline phases are formed in the same sequence and with similar structures in the polyelectrolyte / surfactant system as inpure binary surfactant/ water systems.The order of the phases is as a function of increasing hydrocarbon chain length of the surfactant : un ordered micellar, cubic, hexagonal, cubic and lamellar. Increasing the temperature and decreasing the charge density of the polymer has similar effect, but in this case order of the phases

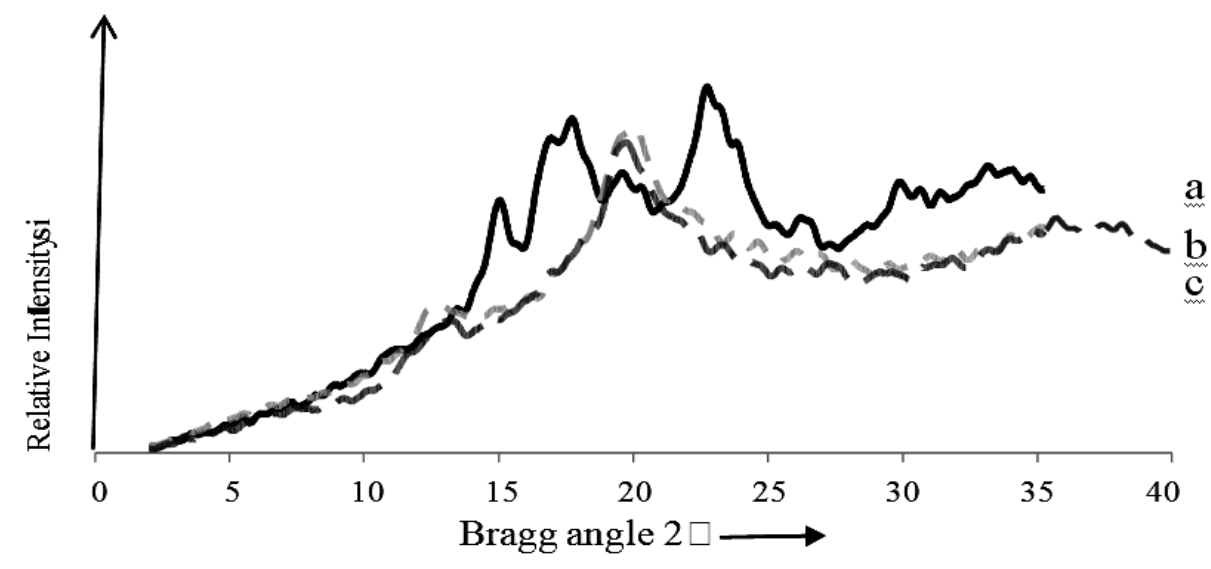

Fig.1: Calligraphy of X-ray diffraction patterns of, $a=$ normal potato starch (black), b=starch with CTAB (mazenta) and c= starch with SDS (green)

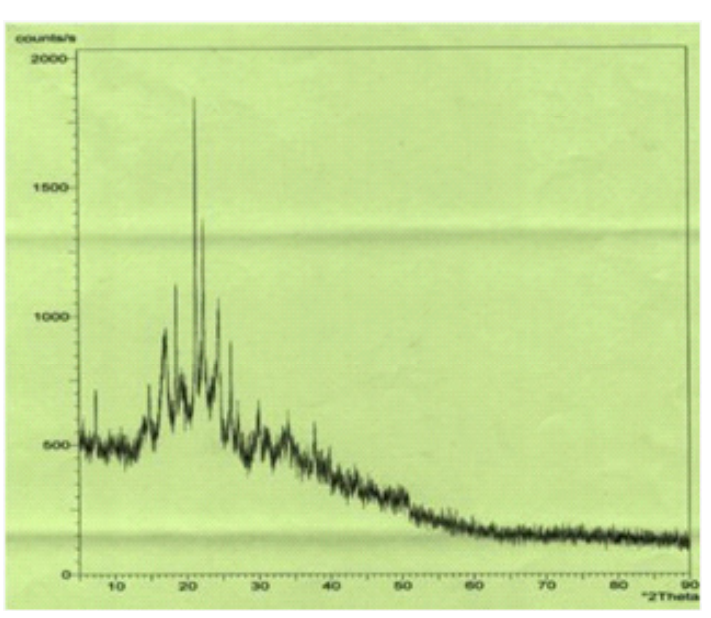

Fig. 2: Only Starch XRD of $2 \theta\left(90^{\circ}\right)$

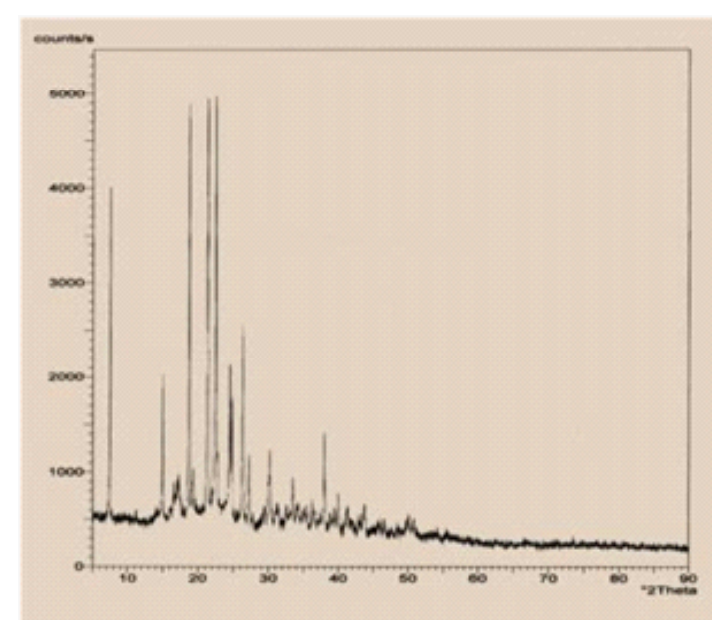

Fig.3: Starch - SDSc omplexes XRD of $2 \theta\left(90^{\circ}\right)$ 
is the opposite : lamellar, cubic, hexagonal, cubic and micellar solution. However, the concentration of surfactant in the poly electrolyte-containing phases is substantially lower than in pure surfactant system and they also precipitate from solutions containing much less surfactant than in the binary system; i.e., the two-phase regions between liquid crystalline phase and solution are very wide. This shows that the effect of adding poly electrolyte is not only an increase in the ionic strength but probably also an increase indirect binding of the poly electrolyte to the aggregate surfaces, which reduces the electrostatic repulsion between the aggregates. This not ion is further substantiated by the discussion below. The structural force, due to the collisions between the molten hydrocarbon chains, creates an outward pressure, tending to expand the hydrocarbon moiety. This is clearly reflected in the temperature dependence of the curvature of the aggregate

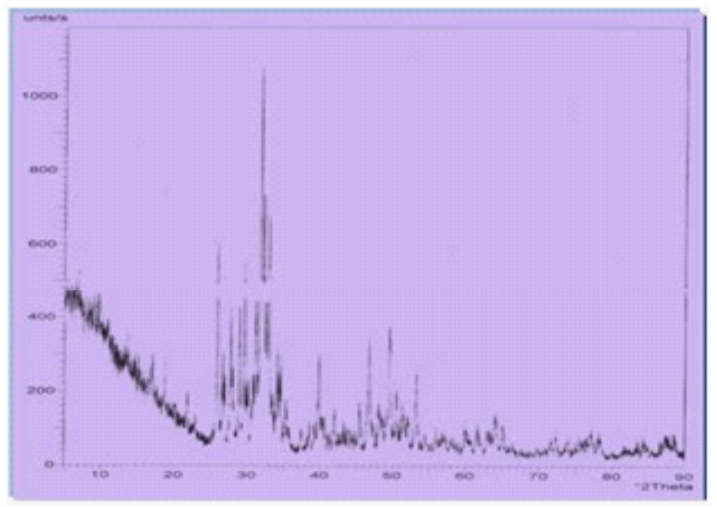

Fig. 4: Starch - CTAB complexes XRD of $2 \theta\left(90^{\circ}\right)$

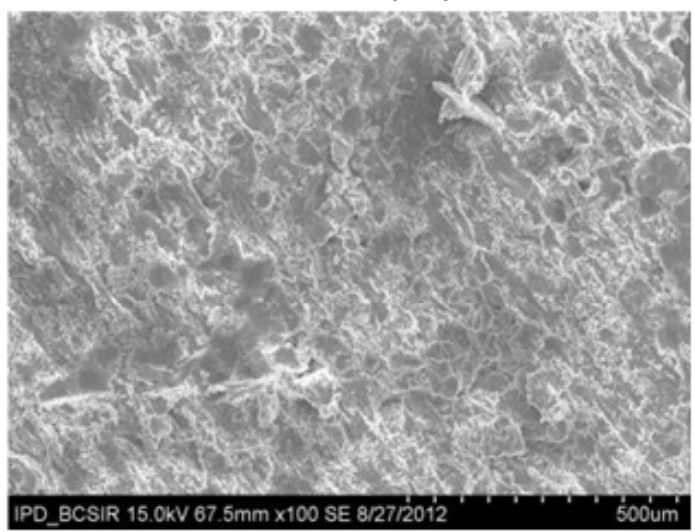

Fig. 6: Only SDS SEM of magnification 100 times in area of $\mathbf{5 0 0}$ micrometer structures both in pure surfactant/water and starch / surfactant / water systems.

At the polar/non-polarinterface, hydrophobic interactions create an inward pressure that tends to reduce water - oil contact. In the head group region the rearesteric, electrostatic, and hydration forces which are expected to createanet positive out ward pressure. When the out ward pressure in the chain region is high the monolayer is likely to bend toward water. On basis of presently available results, theimportance of hydrophobic, steric and hydration forces in the starch /surfactant / water systems are difficult to assess. However, the strong interaction between the sulfate and CTAB groups in the starch/ SDS complexes leads to formation of aggregates with lower curvature than in corresponding starch/ CTAB systems, which indicates that reduction of hydrophobic interactions and salvation in the surface

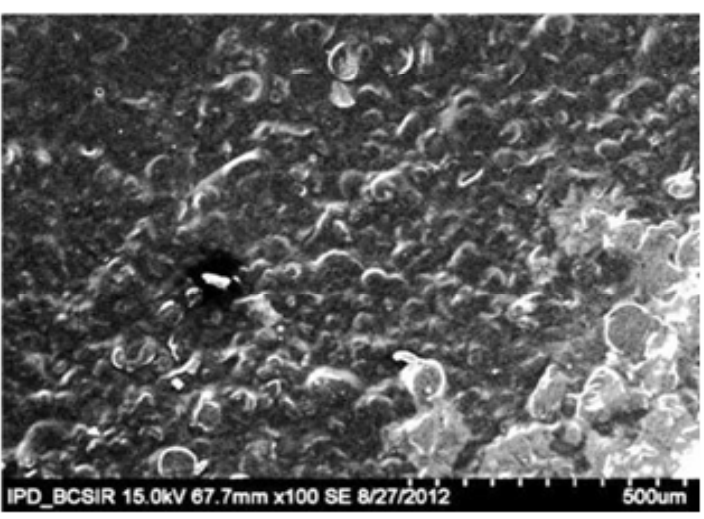

Fig. 5: Only Starch SEM of magnification 100 times in area of 500 micrometer

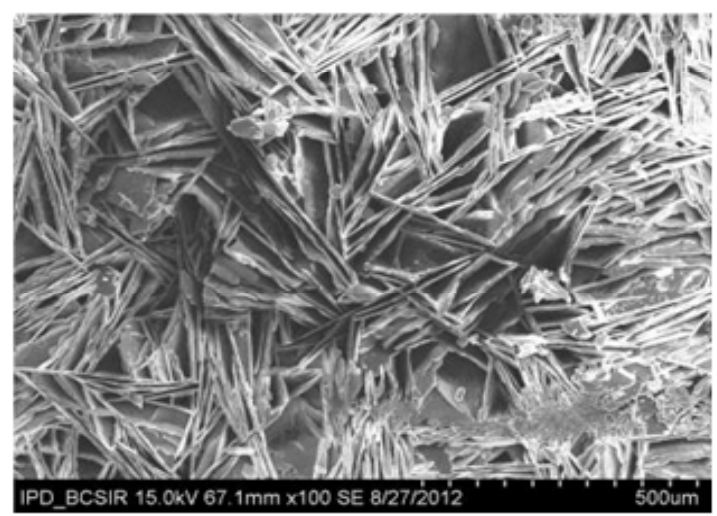

Fig. 7: Starch (amylose)-SDS complexes reacted SEM of magnification 100 times in area of 500 micrometer 
plays a role. Whentheionic strength increases, the phase boundaries of the lyotropic liquidcry stalline phases move toward lower surfactant concentrations. They also shift to higher temperatures, indicating increased stability of the surfactant aggregates. However, this effect is much weaker than the effect of adding a polyelectrolyte. For instance,the phase diagram of the $\mathrm{NaPal} / \mathrm{NaCl} /$ water system. Laughlin ${ }^{40}$ shows that a ddition of upto 5 wt $\%$ of

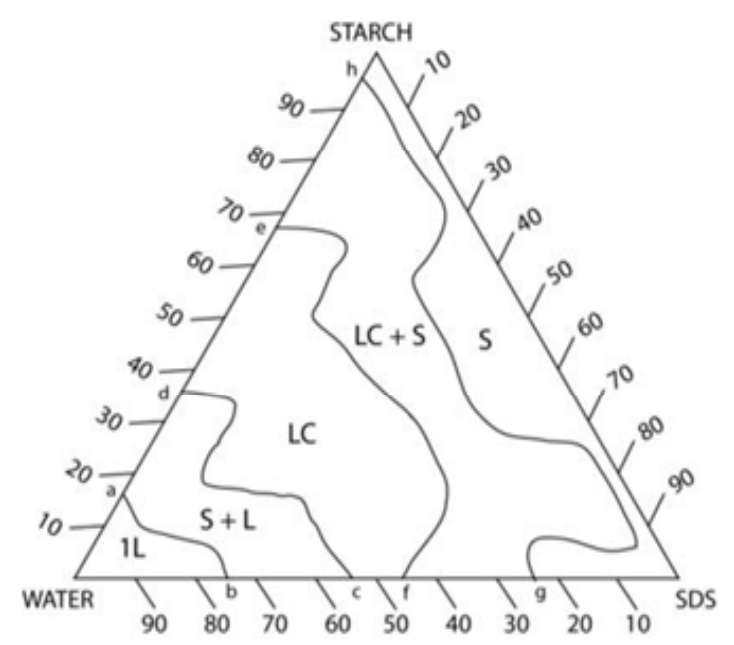

NaCltoa 1 wt\% NaP al solution does not causeany phase changes. In our investigation, the initial SDS concentration of the starch / SDS sample was 1.06 wt $\%$ and the starch concentration was $5 w t \%$. The estimated effect of starch on the ionic strength corresponds approximately to $0.1 \mathrm{wt} \%$ of added SDS. This indicates very clearly that the addition of a polyelectrolyte has a much stronge reffect than the addition of a simple electrolyte.

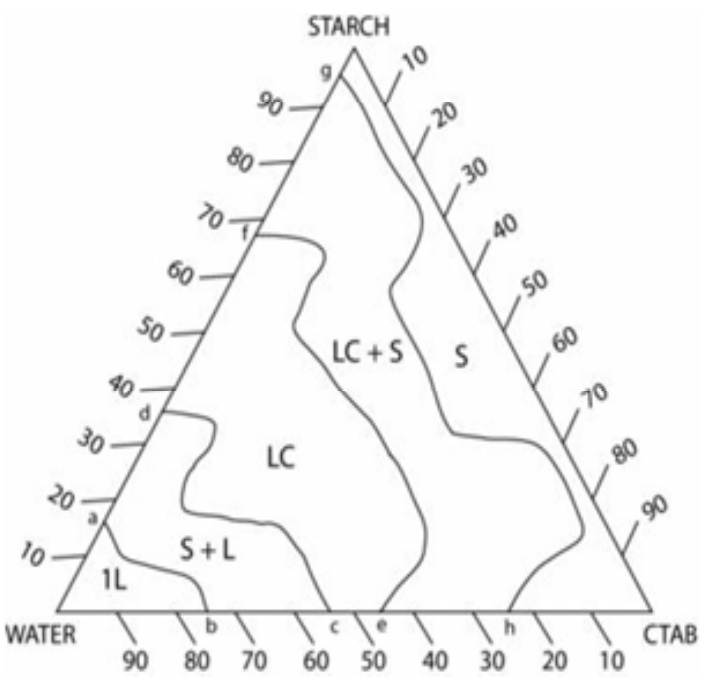

Fig. 8: Ternary phase diagram of (a) Starch (DS 0.80)/SDS/water, (b) Starch (DS 0.80)/water/ CTAB systems at $60^{\circ} \mathrm{C}$. The area in between the points dcef of both Figures described the compositions prepared in experiments of liquid crystal. Here is $1 \mathrm{~L}$, liquid; $\mathrm{S}+\mathrm{L}$, solid+liquid;LC,liquid-crystal;LC+S, liquid crystal + solid; and S solid

\section{CONCLUSION}

Though, starch itself surface inactive but when it is added to ionic surfactants as a soap filler the physical properties of polymeric starch, such as surface tension, reduced viscosity, electric conductance etc. have been changed. It may occurred due to the formation of intermolecular $\mathrm{H}$-bonding through polar part of ionic surfactants (CTAB, SDS) and $\mathrm{H}$-atom from hydroxyl radical of amylase part of starch molecule, whereas non ionic surfactant (Tween-20) is absent. The fact is supported by XRD, SEM and FT-IR instrumental analyses.

\section{ACKNOWLEDGEMENTS}

One of the authors was awarded byNSICT fellowship from Ministry of National Science \& Information and Communication Technology (NSICT), Bangladesh in2008-2011.

\section{REFERENCES}

1. Hayakawa, K. and Kwak,J.C.T.,In Cationic Surfactants (2nded.),Rubingh,N. D., Holland, P. M., (Eds.), Marcel Dekker: NewYork, pp. 189-248 (1991).
2. Robb,I. D.,In Anionic Surfactants-Physical Chemistry of Surfactant Action, LucassenReynders,E. (Ed.), Marcel Dekker: New York, 109-142 (1981). 
3. Saito,S.,In Nonionic Surfactants, Schick,M.J. (Ed.)Marcel Dekker: NewYork, pp.881-926 (1991).

4. Goddard,E. D., Ananthapadmanadhan, K. P. (Eds.), Interactions of Surfactants with Polymers and Proteins, CRCPress: ocaRaton, FL, pp.1-427 (1993).

5. Ramakant Sharma, Orient J. Chem., 29(3): 1199-1202 (2013).

6. Harada, A., Nozakura, S.,Polym.Bull.,11: 175-178 (1984).

7. Chen, L., Yu, S., Kagami, Y., Gong, J., Osada, Y., Macromolecules, 31: 787-794 (1998).

8. Kim,R., Ishizawa, M.,Gong, J., Osada, Y., J. Polym. Sci. A, 37: 635-644 (1999).

9. Kabanov, V.A., Bakeev, K.N., Yang, M.S., Mac Knight, W.J., Zezin, A. B., Macromolecules, 27: 300 (1994).

10. Khandurina,Y.V., Rogacheva, V.B., Zezin, A.B., Kabanov, V.A., J.Polymer. Sci., 36: 184188 (1994).

11. Dembo, A.T., Khandurina, Y.V., Rogacheva, V.B., Zezin, A.B., Kabanov, V.A., J.Polymer. Sci. 36: 189-194 (1994).

12. Rogacheva, V.B.,Khandurina,Y.V.,Zezin, A.B., Kabanov V.A., J. Polymer. Sci., 36: 195-199 (1994).

13. Hansson, P., Langmuir, 14: 4059-4064 (1998).

14. Ilekti, P., Piculell,L., Tounilhac, F., Cabane, B., J. Phys. Chem. B, 102: 344-351 (1998).

15. Kosmella, S., Ko"tz, J., Friberg, S.E., MacKay, R., Colloids Surf.A, 112: 227-231 (1996).

16. Ruppelt, D., Ko"tz, J., Jaeger, W., Friberg, S.E., MacKay, R.,Langmuir,13: 3316-3319 (1997).

17. Zhou, S., Burger, C., Yeh, F., Chu, B., Macromolecules, 31: 8157-8163 (1998).

18. Zhou,S., Yeh, F., Burger, C., Chu, B., J.Phys. Chem. B.,103: 2107-2112 (1999).

19. Merta,J.,Stenius, P., Colloids Surf. A: PhysicochemAsp.,122: 243-255 (1997).

20. Goddard,E.D.,Hannan, R.B., J.Colloid Interface Sci., 55: 73-79 (1976).

21. Goddard,E.D.,Hannan,R.B.,J.Am.OilChem. Soc., 54: 561-566 (1977).

22. Leharman, L., The nature of the fatty acid associated with starch. The adsorption of palmitic acid by potato and defatted corn and rice starches, J. Am. Chem. Soc.,64: 2144
(1942).

23. Whistler, R.L., and Hilburt, G.E., Extraction of fatty substance from starch,J. Am. Chem. Soc.,66: 1721 (1944).

24. Zobel,H. F.,X-ray analysis of starchgranules. In Methods in Carbo-hydrate Chemistry (Vol. 4),Whistler, R. L. (ed.), Academic Press: NewYork,NY, pp.109-113 (1964)

25. Osman, E. M., Leith, S. J., andFles, M., Complexes of amylase with surfactants, Cereal Chem., 38: 449 (1961).

26. Krog, N. and Nybo-Jensen, B., Interaction of monoglycerides in different physical states with amylase and their anti-firming effects in bread, J. Food Technol.,5, 77 (1970).

27. Atichokudomchai, N., Shobsngob, S. and Varavinit, S., Morphological properties of acid- modified tapiocastarch, Starch/Starke, 52: 283-289 (2000).

28. Blazek, J. and, Copeland, L., The effect of monopalmitin on pasting properties of wheat starches with varyingamylosecontent, Carbohydrate Polymers, 78: 131-133 (2009).

29. Ghiasi, K., Varriano-Marston, E. and Hoseney, R. C., Gelatinization of wheat starchll: StarchSurfact antinteraction, Cereal Chemistry, 59(2): 86-88 (1982).

30. Tang, M.C. and Copeland, L., Analysis of complexes between lipids and wheat starch, Carbohydrate Polymers, 67: 80-85 (2007).

31. Copeland, L., Blazek, J., Salman, H and Tang, M. C., Form and functionality of starch, Food Hydrocolloids, 23: 1527-1534 (2009).

32. Abdel-Aal,E. S. M., Hucl,P.,Chibbar,R. N., Han,H. L., andDemeke,T., Physiochemical and structural characteristics of flours and starches from waxy and non waxy wheats, Cereal Chemistry,79(3): 458-464 (2002).

33. Banks, W. and Greenwood, C.T., In Starch and Its Components, Halstead press: Division of John Wileyand Sons, Inc., New York, 67-110 (1975).

34. Kainuma, K. and French, D., Nagelia mylodextrin and its relationship to starch granule structure. . Preparation and properties of amylodextrins from various starchtypes, Biopolymers, 10: 1673 (1971).

35. Robin, J. P., Mercier, C., Charbonniere, R.andGuilbot, A., In soluble residues from 
prolonged and treatment of potato starch, Cereal Chemistry, 51: 389 (1974).

36. Sarko, A. and Wu, H. C. H., The crystal structures of A-B-and C polymorphos of amylose, Starch/ Starke, 30: 73-78 (1978).

37. Caesar,G. V., and Cushing, M. L., The starchmolecule, Journal of physical chemistry, 45: 776 (1971).

38. Gray, V. M., and Schoch, J. J., Effects of surfactants and fatty adjuncts on the swelling and solubilization on granular, Starch/ Starke, 14: 239 (1962).

39. Yamashita, Y., Single crystals of amyloseV complexes, Journal of polymer Science, A3: 3251 (1965).

40. Laughlin, R.G., The Aqueous Phase Behaviour of Surfactants, Academic Press: NewYork, 588-597 (1994). 